\title{
Extracorporeal Lung Support for Hypercapnic Ventilatory Failure
}

\author{
Lara Pisani PhD, Massimiliano Polastri MSc, Angela Maria Grazia Pacilli MD, and \\ Stefano Nava MD
}

\author{
Introduction \\ Extracorporeal Lung Support as a Bridge to Lung Transplantation \\ Extracorporeal Lung Support in Respiratory Diseases \\ Severe Asthma \\ Acute Interstitial Lung Disease \\ COPD \\ Rehabilitation While Receiving Extracorporeal Lung Support \\ Summary
}

\begin{abstract}
Extracorporeal lung support can be achieved using extracorporeal membrane oxygenation (ECMO) and extracorporeal $\mathrm{CO}_{2}$ removal. The ECMO systems allow a total lung support, providing both blood oxygenation and $\mathrm{CO}_{2}$ removal. Unlike ECMO, extracorporeal $\mathrm{CO}_{2}$ removal refers to an extracorporeal circuit that provides a partial lung support and selectively extracts $\mathrm{CO}_{2}$ from blood. The concept of partial extracorporeal lung support by removing only $\mathrm{CO}_{2}$ without effect on oxygenation was first proposed in 1977 by Kolobow and Gattinoni, with the aim to reduce breathing frequency, ventilator tidal volumes, and inspiratory pressures, facilitating lung-protective ventilation. Patients with end-stage chronic lung disease can survive, while waiting for lung transplantation, only if treated with mechanical ventilation or extracorporeal lung support. ECMO has been considered a suitable approach as a bridge to lung transplantation for patients with advanced respiratory failure waiting for lung transplantation. Extracorporeal $\mathrm{CO}_{2}$ removal has been proposed for the treatment of COPD patients suffering from exacerbation to avoid invasive mechanical ventilation. The rationale is to combine the improvement of alveolar ventilation by using noninvasive ventilation with muscle unload provided by removing $\mathrm{CO}_{2}$ directly from the blood, using an extracorporeal device. Increasing attention has been given to the possibility of patients performing a variety of physical activities while receiving extracorporeal lung support. This is possible thanks to the continuous development of technology together with the customization of sedative protocols. Awake extracorporeal support is a specific approach in which the patient is awake and potentially cooperative while receiving ECMO. The present analysis aims to synthesize the main results obtained by using extracorporeal circuits in patients with respiratory failure, particularly in those patients with hypercapnia. Key words: extracorporeal membrane oxygenation; physiotherapy; respiratory insufficiency; critical illness; lung transplantation; survival; $\mathrm{CO}_{2}$. [Respir Care 2018;63(9):1174-1179. (C) 2018 Daedalus Enterprises]
\end{abstract}

\section{Introduction}

Extracorporeal lung support can be achieved using extracorporeal membrane oxygenation (ECMO) and extracorporeal $\mathrm{CO}_{2}$ removal. ${ }^{1}$ The ECMO systems allow a total lung support, providing both blood oxygenation and $\mathrm{CO}_{2}$ removal. Blood flow and the oxygen-carrying capacity, which is dependent on the hemoglobin concentration and on the venous blood oxyhemoglobin saturation, control oxygenation. ${ }^{2}$ In contrast, $\mathrm{CO}_{2}$ removal depends not only 
on the blood flow but also on the gas flow through the membrane (sweep gas). ${ }^{2}$ Moreover, ECMO devices could also provide adequate hemodynamic support in patients suffering from both cardiac and pulmonary failure. Unlike ECMO, extracorporeal $\mathrm{CO}_{2}$ removal refers to an extracorporeal circuit that provides a partial lung support and selectively extracts $\mathrm{CO}_{2}$ from blood. ${ }^{3}$

The concept of partial extracorporeal lung support by removing only $\mathrm{CO}_{2}$ without effect on oxygenation was first proposed in 1977 by Kolobow and Gattinoni, ${ }^{4}$ with the aim to reduce breathing frequency, ventilator tidal volumes, and inspiratory pressures, facilitating lung-protective ventilation. Extracorporeal $\mathrm{CO}_{2}$ removal devices require a minimally invasive approach and present many advantages compared with conventional ECMO systems, including a lower blood flow (range from 300 up to $1,500 \mathrm{~mL} / \mathrm{min}$ vs $5,000 \mathrm{~mL} / \mathrm{min}$ ). ${ }^{3,5}$ This is due to the physical dissolving of $\mathrm{CO}_{2}$ and its better diffusion capacity than that of hemoglobin-bound oxygen. Because of the lower blood flows, the cannula size is smaller (12-14 French), causing less vascular trauma. Continuous infusion of heparin is still needed to prevent clotting of the circuit.

In the last decade, some important technical issues have been pointed out also in an attempt to ameliorate the performance and ease of use of extracorporeal $\mathrm{CO}_{2}$ removal devices, such as the importance of large artificial lung surface and the use of acidification in the circuit to achieve a better $\mathrm{CO}_{2}$ removal. ${ }^{6,7}$ In addition, a respiratory electrodialysis has been developed that selectively modulates $\mathrm{pH}$ and electrolyte concentration and greatly enhances $\mathrm{CO}_{2}$ removal by applying an electrical field to blood. ${ }^{8}$ The present analysis aims to synthesize the main results obtained by using extracorporeal circuits in patients with respiratory failure, particularly in those patients with hypercapnia.

\footnotetext{
Dr Pisani is affiliated with the Respiratory and Critical Care Unit, Department of Clinical, Integrated, and Experimental Medicine (DIMES), and Mr Polastri is affiliated with Physical Medicine and Rehabilitation, Medical Department of Continuity of Care and Disability, University Hospital St. Orsola-Malpighi, Bologna, Italy. Drs Pacilli and Nava are affiliated with the School of Medicine, Alma Mater Studiorum University of Bologna, Bologna, Italy.
}

Dr Nava has disclosed relationships with ESTOR, Hemodec, and ALung Technologies. The other authors have disclosed no conflicts of interest.

Dr Nava presented a version of this paper at the 33rd annual New Horizon Symposium at the AARC Congress 2017, held October 4-7, 2017, in Indianapolis, Indiana.

Correspondence: Stefano Nava MD, Department of Clinical, Integrated and Experimental Medicine (DIMES), School of Medicine, Alma Mater Studiorum University of Bologna, Via G Massarenti 9, 40138 Bologna, Italy. E-mail: stefano.nava@aosp.bo.it.

DOI: $10.4187 /$ respcare.06277

\section{Extracorporeal Lung Support as a Bridge to Lung Transplantation}

Patients with end-stage lung failure due to a chronic respiratory disease can survive, while waiting for lung transplantation, only if treated with mechanical ventilation or extracorporeal lung support. ECMO has been considered a suitable approach as a bridge to lung transplantation for patients with advanced respiratory failure waiting for lung transplantation. ${ }^{9}$ The possibility of maintaining patients awake and interactive while they receive extracorporeal support (awake ECMO) has increased the diffusion of this practice in end-stage respiratory failure disorders, such as COPD, cystic fibrosis, pulmonary fibrosis, pulmonary arterial hypertension, bronchiolitis obliterans syndrome, and sarcoidosis. 9 A systematic review described data from 14 studies, including 441 subjects with endstage diseases undergoing ECMO as a bridge to transplantation. Post-transplant survival was similar when comparing data from mechanically ventilated and ECMO-bridged subjects. ${ }^{9}$ In this group, mortality before transplantation ranged between 17 and 50\%. Among the factors that can affect mortality, the most frequent are multiple organ failure, sepsis, cardiac failure, and bleeding. One-year survival post-transplantation ranged between 50 and $90 \%$. Contraindications to ECMO as a bridge option have been identified in sepsis, neurological impairments, severe malnutrition, and severe graft dysfunction, regardless of patient age.

To enhance ECMO outcomes in bridged-to-transplant patients, noninvasive ventilation (NIV) has been recognized as an alternative to mechanical ventilation with the aim to prevent ventilator-associated complications. Endotracheal intubation in patients with end-stage lung disease waiting for lung transplantation is associated with a poor outcome. ${ }^{10}$ In contrast to ECMO, which has been reported in several studies as a successful bridging technique in patients with primarily hypoxic terminal respiratory failure, the use of extracorporeal $\mathrm{CO}_{2}$ removal as a bridge to lung transplant in patients with primarily hypercapnic terminal insufficiency is still limited. ${ }^{11}$ The largest series, which described the use of extracorporeal $\mathrm{CO}_{2}$ removal as a bridge to lung transplant in a total of 20 subjects with hypercapnic respiratory failure, was reported by Schellongowski et al. ${ }^{11}$ Two different extracorporeal $\mathrm{CO}_{2}$ removal devices were used; 10 subjects were supported by a pumpdriven venovenous iLA (interventional lung assist) active system and the others by the pumpless arteriovenous Novalung iLA device (ILA, Novalung, Heilbronn, Germany). No difference in terms of $\mathrm{CO}_{2}$ reduction was found. Four subjects were switched to full ECMO because of persistent hypoxemia and/or additional cardiac failure. Nineteen subjects $(95 \%)$ were successfully transplanted. The overall survival at $1 \mathrm{y}$ was $72 \% .{ }^{11}$ However, given the paucity of 
evidence, the efficacy of extracorporeal $\mathrm{CO}_{2}$ removal as a bridge to lung transplantation in addition, or as an alternative, to mechanical ventilation remains controversial.

\section{Extracorporeal Lung Support in Respiratory Diseases}

\section{Severe Asthma}

Near-fatal asthma is a life-threatening subset of asthma, sometimes requiring mechanical ventilation..$^{12}$ Because of air-flow obstruction with hyperinflation and the development of intrapulmonary shunt, often caused by atelectasis and mucous plugging, patients with severe asthma frequently show a rapid onset with severe hypoxemia as well as a persistent hypercapnia. ${ }^{12}$ Other relevant pathophysiologic events include lactic acidosis with signs of exhaustion, worsening of mental status, and hemodynamic instability. ${ }^{12}$ Despite the fact that NIV, together with conventional medical treatment, decreases respiratory muscle work and improves alveolar ventilation, it is not recommended for patients with acute severe asthma due to the lack of evidence. ${ }^{13}$ In addition, intubation in this scenario could be challenging and not without risks. Therefore, extracorporeal lung support has been considered a valid option in the management of patients with severe asthma to avoid sedation and intubation and their negative effects. In the case of a hypercapnic pediatric patient, the use of ECMO has been described as a viable therapeutic option to treat hypercapnic respiratory failure accompanied by a left-lung atelectasis. ${ }^{14}$ Recently, Schneider et al ${ }^{15}$ demonstrated that extracorporeal $\mathrm{CO}_{2}$ removal was an effective tool for management of severe respiratory acidosis in a patient with a near-fatal asthma attack who was failing NIV. Following resolution of the acute attack, after $34 \mathrm{~h}$ of extracorporeal $\mathrm{CO}_{2}$ removal treatment, the patient was completely weaned without any complication.

\section{Acute Interstitial Lung Disease}

Idiopathic interstitial pneumonias are a group of heterogeneous lung diseases with unknown etiology. The case reported by Petzoldt et al ${ }^{16}$ was affected by nonspecific interstitial pneumonia, the first line treatment for which is high-dose corticosteroids. The authors described the successful management of a patient with severe refractory hypercapnia and signs of encephalopathy using the arteriovenous pumpless extracorporeal lung assist device (Novalung, Talheim, Germany) as rescue therapy. ${ }^{16}$ Because of the poor prognosis of other forms of idiopathic interstitial pneumonias, such as the idiopathic pulmonary fibrosis, the use of extracorporeal $\mathrm{CO}_{2}$ removal should be limited to potentially reversible conditions or to use as a transplantation bridge.

\section{COPD}

Respiratory support is often needed in patients with severe COPD exacerbation, and NIV is currently considered the preferred choice for these patients when acute hypercapnic respiratory failure occurs. ${ }^{13}$ Although NIV reduces mortality, the need for endotracheal intubation, and the length of hospital stay in these patients, ${ }^{13}$ it can fail. In addition, COPD patients who require intubation after NIV failure usually have a poor outcome with high mortality rates. ${ }^{17}$ Moreover, NIV is not indicated in patients with cardiac and respiratory arrest, and it could be difficult to apply when patients are agitated, unable to protect the airway, and unable to manage excessive secretions. ${ }^{18}$ Thus, extracorporeal $\mathrm{CO}_{2}$ removal has been proposed for the treatment of patients suffering a COPD exacerbation to avoid invasive mechanical ventilation. The rationale is to combine the improvement of alveolar ventilation by using NIV with muscle unloading provided by removing $\mathrm{CO}_{2}$ directly from the blood, using an extracorporeal device. Similarly, a physiologic basis for the application of extracorporeal $\mathrm{CO}_{2}$ removal to accelerate the weaning process from the endotracheal intubation has been demonstrated. ${ }^{19,20}$ In fact, extracorporeal $\mathrm{CO}_{2}$ removal was able to reduce inspiratory effort, avoiding the shallow-breathing pattern and maintaining stable $\mathrm{P}_{\mathrm{aCO}}$ levels when applied during T-piece weaning trial in severely hypercapnic COPD subjects previously intubated for an episode of respiratory acidosis. ${ }^{19}$

Although extracorporeal low-blood flow devices are extremely efficient from a physiologic standpoint, scientific evidence is still limited. The majority of data are from case reports and observational studies, one of them quite large but performed in different groups of subjects (ie, those receiving NIV with a high likelihood of requiring invasive ventilation, those who could not be weaned from NIV, and finally those receiving invasive ventilation who had failed attempts to wean). ${ }^{21}$ Randomized controlled studies have never been done. There have been 3 case-control studies published to date. In a retrospective study using a pumpless extracorporeal lung assist, 21 cases were matched with contemporaneous controls (only one fourth of the sites) based on diagnosis, age, Simplified Acute Physiology Score II, and $\mathrm{pH} \pm 0.05$ before extracorporeal $\mathrm{CO}_{2}$ removal or intubation. Nineteen $(90 \%)$ of the 21 subjects treated with pumpless extracorporeal lung assist did not require intubation. ${ }^{22}$ Median $\mathrm{P}_{\mathrm{aCO}_{2}}$ levels and $\mathrm{pH}$ in arterial blood before treatment were $84 \mathrm{~mm} \mathrm{Hg}$ and 7.28, respectively; within $24 \mathrm{~h}$, median $\mathrm{P}_{\mathrm{aCO}}$ levels and $\mathrm{pH}$ had significantly improved to $52.1 \mathrm{~mm} \mathrm{Hg}$ and 7.44 .

Twenty-five subjects considered at risk of NIV failure were treated with NIV plus extracorporeal $\mathrm{CO}_{2}$ removal 
by Del Sorbo et al. ${ }^{23}$ NIV criteria for failure were identified as the presence of 2 or more of the following conditions for a duration of at least $2 \mathrm{~h}$ : (1) frequency $>30$ breaths/min, (2) arterial $\mathrm{pH} \leq 7.30$, (3) $\mathrm{P}_{\mathrm{aCO}_{2}}>20 \%$ of the baseline value, and (4) use of accessory muscles or paradoxical abdominal movements. Compared with 21 matched NIV historical control subjects, the adjunction of extracorporeal $\mathrm{CO}_{2}$ removal to NIV was able to reduce acutely $\mathrm{CO}_{2}$ levels, decreasing the relative risk of intubation by $73 \%$ (hazard ratio $0.27,95 \%$ CI $0.07-0.98, P=.047$ ). However, relevant extracorporeal $\mathrm{CO}_{2}$ removal-associated adverse events were observed in 13 subjects (52\%). ${ }^{23} \mathrm{~A}$ systematic review assessed the efficacy and safety of extracorporeal $\mathrm{CO}_{2}$ removal in 87 subjects with hypercapnic respiratory failure across 10 different studies, showing heterogeneous data. ${ }^{3}$ Sources of heterogeneity included the subjects' characteristics, the types of extracorporeal $\mathrm{CO}_{2}$ removal devices, the anticoagulation protocols, and the sizes of the vascular access. Despite these limitations, the majority of subjects in this review successfully were sustained on NIV and avoided intubation (93\%); furthermore, more than half of the subjects were weaned from mechanical ventilation (53\%). These high success rates in the primary outcomes, however, must be interpreted very cautiously, given the clear selection bias associated with case series data. Besides, all of the studies were associated with a high rate of complications; a total of 11 major and 30 minor complications were found.

Only 3 studies presented data on ICU and hospital stay and mortality, and they proved to be contrasting. ${ }^{3}$ The available physiologic data, comparing primarily pre- with post-extracorporeal $\mathrm{CO}_{2}$ removal changes in the same patients, suggest rapid and sustained improvements in ventilatory parameters, including $\mathrm{pH}, \mathrm{P}_{\mathrm{aCO}}$, and breathing frequency, but not in oxygenation as measured using $\mathrm{P}_{\mathrm{aO}_{2}} / \mathrm{F}_{\mathrm{IO}_{2}}$. Another multi-center case-control study 24 showed that intubation was avoided in 14 of 25 extracorporeal $\mathrm{CO}_{2}$ removal subjects (56\%). Once again, relevant extracorporeal $\mathrm{CO}_{2}$ removal-associated adverse events were observed in over one third of cases. Last, despite the shorter period of invasive mechanical ventilation in the extracorporeal $\mathrm{CO}_{2}$ removal group, there were no significant differences in stay or in 28-d mortality (16\% vs $12 \%)$ and 90 -d mortality (28\% vs $28 \%$ ) rates between the 2 groups. In conclusion, the topic appears to be clinically relevant and innovative. These data provide the basis for randomized clinical trials needed to assess the risk-benefit balance of extracorporeal $\mathrm{CO}_{2}$ removal in COPD exacerbations. In addition, further investigations are needed to elucidate more clearly the cost-effectiveness as well as ethical implications of this treatment.

\section{Rehabilitation While Receiving Extracorporeal Lung Support}

Increasing attention has been given to the possibility of patients performing a variety of physical activities while receiving extracorporeal lung support. This is attainable thanks to the continuous development of technology together with the customization of sedative protocols. Awake extracorporeal support is a specific approach in which the patient is awake and potentially cooperative while receiving ECMO. A systematic review found that ambulation was possible in patients awaiting lung transplantation during awake venovenous ECMO. Both passive or active range-of-motion exercises, postural transfers, and in-bed positioning have been found to be major interventions carried out during venovenous ECMO support, usually starting within the first $2-5 \mathrm{~d}$ of treatment. ${ }^{25} \mathrm{~A}$ recent consensus agreement provided best-practice evidence for the provision of rehabilitation in patients receiving ECMO support. This document has been recently released with the endorsement of the Association of Chartered Physiotherapists in Respiratory Care Committee. ${ }^{26}$ It highlighted the importance of a multidisciplinary approach to ECMO patients, and, for the first time in the literature, described detailed procedures related to the rehabilitation treatment in such patients.

Decannulation is a potential life-threatening risk, and a specialized team should be trained to provide mobilization in ECMO patients. With reference to the rehabilitation procedures, patient safety must be guaranteed by planning an appropriate pretreatment evaluation and by recognizing that any team member has the authority to interrupt the progression of physiotherapy treatment in the presence of any concern. In addition, mobilization should be performed by at least 2 clinicians. ${ }^{26}$ Thus, major indications for physiotherapy can be summarized by the following objectives: prevention of bed-related complications, improvement of functional ability and mood restoration, optimization of an early recovery, and improvement of clearance of secretions, and pulmonary ventilation. ${ }^{26}$ Whereas an increasing number of experiences have been published regarding rehabilitation and ECMO support, less evidence has been found on physiotherapeutic treatment in patients receiving extracorporeal $\mathrm{CO}_{2}$ removal. In a pilot study, active daily rehabilitation was provided in 5 subjects with COPD who underwent $\mathrm{CO}_{2}$ removal to facilitate extubation by using upper-body venous configuration via a dual lumen cannula with the goal of daily ambulation while in the ICU. ${ }^{20}$

\section{Summary}

We have reviewed what is known about extracorporeal lung support for ventilatory failure. What emerged was that the management of patients undergoing extracorpo- 
real lung support is a multidisciplinary matter involving several professions, including respiratory therapists, thoracic surgeons, physiotherapists, nurses, and intensivists. $20,25,26$ The creation of an international registry in 1989 by the Extracorporeal Life Support Organization has contributed to collecting data from 75,000 ECMO patients, enhancing the knowledge of extracorporeal life support procedures. ${ }^{27}$ Together with the Extracorporeal Life Support Organization, the International ECMO Network is contributing to developing best practices for the organization of ECMO procedures. ${ }^{27}$ In addition, from the available data, it appears that the safety of patients receiving venovenous $\mathrm{ECMO}$ is the major concern for professionals involved in the physiotherapeutic intervention. ${ }^{25,26,28}$ Last, there is another important aspect that needs to be underlined. The development of national networks of rehabilitative interest for ECMO patients has provided indications for a multidisciplinary approach in such a particular class of patients. ${ }^{26}$ This will represent in the near future an area of great development worthy of further investigation. Extracorporeal $\mathrm{CO}_{2}$ removal is today a consolidated technique that can help patients to survive life-threatening situations. In particular, extracorporeal $\mathrm{CO}_{2}$ removal can contribute to avoiding endotracheal intubation in COPD patients at risk of failure or failing NIV and can be a rescue therapy (bridge to lung transplantation) in severe hypercapnia. Preliminary physiologic studies show that extracorporeal $\mathrm{CO}_{2}$ removal may also reduce respiratory distress during a weaning trial, suggesting that it may be appropriate in the weaning process. Extracorporeal $\mathrm{CO}_{2}$ removal should be performed within an environment with appropriate staff allocation.

\section{REFERENCES}

1. Braune SA, Kluge S. Extracorporeal lung support in patients with chronic obstructive pulmonary disease. Minerva Anestesiol 2013; 79(8):934-943.

2. Bartlett RH. Physiology of gas exchange during ECMO for respiratory failure. J Intensive Care Med 2017;32(4):243-248.

3. Sklar MC, Beloncle F, Katsios CM, Brochard L, Friedrich JO. Extracorporeal carbon dioxide removal in patients with chronic obstructive pulmonary disease: a systematic review. Intensive Care Med 2015;41(10):1752-1762.

4. Kolobow T, Gattinoni L, Tomlinson T, White D, Pierce J, Iapichino G. The carbon dioxide membrane lung (CDML): a new concept. Trans Am Soc Artif Intern Organs 1977;23:17-21.

5. de Villiers Hugo J, Sharma AS, Ahmed U, Weerwind PW. Quantification of carbon dioxide removal at low sweep gas and blood flows. J Extra Corpor Technol 2017;49(4):257-261.

6. Karagiannidis C, Strassmann S, Brodie D, Ritter P, Larsson A, Borchardt R, Windisch W. Impact of membrane lung surface area and blood flow on extracorporeal $\mathrm{CO}_{2}$ removal during severe respiratory acidosis. Intensive Care Med Exp 2017;5(1):34.

7. Zanella A, Patroniti N, Isgrò S, Albertini M, Costanzi M, Pirrone F, et al. Blood acidification enhances carbon dioxide removal of membrane lung: an experimental study. Intensive Care Med 2009;35(8): 1484-1487.
8. Zanella A, Castagna L, Salerno D, Scaravilli V, Abd El Aziz El Sayed Deab S, Magni F, et al. Respiratory electrodialysis. A novel, highly efficient extracorporeal $\mathrm{CO}_{2}$ removal technique. Am J Respir Crit Care Med 2015;192(6):719-726.

9. Chiumello D, Coppola S, Froio S, Colombo A, Del Sorbo L. Extracorporeal life support as bridge to lung transplantation: a systematic review. Crit Care 2015;19:19.

10. Fuehner T, Kuehn C, Hadem J, Wiesner O, Gottlieb J, Tudorache I, et al. Extracorporeal membrane oxygenation in awake patients as bridge to lung transplantation. Am J Respir Crit Care Med 2012; 185(7):763-768.

11. Schellongowski P, Riss K, Staudinger T, Ullrich R, Krenn CG, Sitzwohl $\mathrm{C}$, et al. Extracorporeal $\mathrm{CO}_{2}$ removal as bridge to lung transplantation in life-threatening hypercapnia. Transpl Int 2015;28(3): 297-304.

12. Restrepo RD, Peters J. Near-fatal asthma: recognition and management. Curr Opin Pulm Med 2008;14(1):13-23.

13. Rochwerg B, Brochard L, Elliott MW, Hess D, Hill NS, Nava S, et al. Official ERS/ATS clinical practice guidelines: noninvasive ventilation for acute respiratory failure. Eur Respir J 2017;50(2): 1602426 .

14. Tonan M, Hashimoto S, Kimura A, Matsuyama H, Kinose H, Sawada M, et al. Successful treatment of severe asthma-associated plastic bronchitis with extracorporeal membrane oxygenation. J Anesth 2012; 26(2):265-268.

15. Schneider TM, Bence T, Brettner F. "Awake" ECCO2R superseded intubation in a near-fatal asthma attack. J Intensive Care 2017;5:53.

16. Petzoldt M, Braune S, Bittmann I, Kluge S. Rescue therapy with a pumpless extracorporeal lung assist device in a patient with acute interstitial lung disease and severe refractory hypercapnia. Respir Care 2012;57(2):293-297.

17. Chandra D, Stamm JA, Taylor B, Ramos RM, Satterwhite L, Krishnan JA, et al. Outcomes of noninvasive ventilation for acute exacerbations of chronic obstructive pulmonary disease in the United States, 1998-2008. Am J Respir Crit Care Med 2012;185(2):152159.

18. Nava S, Hill N. Non-invasive ventilation in acute respiratory failure. Lancet 2009;374(9685):250-259.

19. Pisani L, Fasano L, Corcione N, Comellini V, Guerrieri A, Ranieri $\mathrm{MV}$, Nava S. Effects of extracorporeal $\mathrm{CO}_{2}$ removal on inspiratory effort and respiratory pattern in patients who fail weaning from mechanical ventilation. Am J Respir Crit Care Med 2015;192(11): 1392-1394.

20. Abrams DC, Brenner K, Burkart KM, Agerstrand CL, Thomashow BM, Bacchetta M, Brodie D. Pilot study of extracorporeal carbon dioxide removal to facilitate extubation and ambulation in exacerbations of chronic obstructive pulmonary disease. Ann Am Thorac Soc 2013;10(4):307-314.

21. Burki NK, Mani RK, Herth FJF, Schmidt W, Teschler H, Bonin F, et al. A novel extracorporeal $\mathrm{CO}_{2}$ removal system: results of a pilot study of hypercapnic respiratory failure in patients with COPD. Chest 2013;143(3):678-686.

22. Kluge S, Braune SA, Engel M, Nierhaus A, Frings D, Ebelt H, et al. Avoiding invasive mechanical ventilation by extracorporeal dioxide removal in patients failing noninvasive ventilation. Intensive Care Med 2012;38(10):1632-1639.

23. Del Sorbo L, Pisani L, Filippini C, Fanelli V, Fasano L, Terragni P, et al. Extracorporeal $\mathrm{CO}_{2}$ removal in hypercapnic patients at risk of noninvasive ventilation failure: a matched cohort study with historical control. Crit Care Med 2015;43(1):120-127.

24. Braune S, Sieweke A, Brettner F, Staudinger T, Joannidis M, Verbrugge $\mathrm{S}$, et al. The feasibility and safety of extracorporeal carbon dioxide removal to avoid intubation in patients with COPD unre- 


\section{ECLS FOR Hypercapnic Ventilatory FaILURE}

sponsive to noninvasive ventilation for acute hypercapnic respiratory failure (ECLAIR study): multicentre case-control study. Intensive Care Med 2016;42(9):1437-1444.

25. Polastri M, Loforte A, Dell'Amore A, Nava S. Physiotherapy for patients on awake extracorporeal membrane oxygenation: a systematic review. Physiother Res Int 2016;21(4):203-209.

26. Eden A, Purkiss C, Cork G, Baddeley A, Morris K, Carey L, et al. In-patient physiotherapy for adults on veno-venous extracorporeal membrane oxygenation - United Kingdom ECMO Physiotherapy
Network: a consensus agreement for best practice. J Intensive Care Soc 2017;18(3):212-220.

27. Combes A, Brodie D, Chen YS, Fan E, Henriques JPS, Hodgson C, et al. The ICM research agenda on extracorporeal life support. Intensive Care Med 2017;43(9):1306-1318.

28. Boling B, Dennis DR, Tribble TA, Rajagopalan N, Hoopes CW. Safety of nurse-led ambulation for patients on venovenous extracorporeal membrane oxygenation. Prog Transplant 2016;26(2): 112-116. 